\title{
Efeitos da terapia de realidade virtual no controle postural de crianças com síndrome
}

\section{de Down}

\author{
Effects of virtual reality therapy on postural control of Down syndrome children \\ Efectos de la terapia de realidad virtual en el control postural de los niños con síndrome de Down
}

Recebido: 10/05/2021 | Revisado: 16/05/2021 | Aceito: 18/05/2021 | Publicado: 04/06/2021

\author{
Lorena Fuchs Silva \\ ORCID: https://orcid.org/ 0000-0001-7162-8557 \\ Universidade Federal do Triângulo Mineiro, Brasil \\ E-mail: lorenafuchs@hotmail.com \\ Janaine Brandão Lage \\ ORCID: https://orcid.org/ 0000-0002-1712-5593 \\ Universidade Federal do Triângulo Mineiro, Brasil \\ E-mail: ja.bl@terra.com.br \\ Luciane Aparecida Pascucci Sande de Souza \\ ORCID: https://orcid.org/ 0000-0002-7160-9556 \\ Universidade Federal do Triângulo Mineiro, Brasil \\ E-mail: lusande@gmail.com \\ Rodrigo César Rosa \\ ORCID: https://orcid.org/ 0000-0003-3157-0826 \\ Universidade Federal do Triângulo Mineiro, Brasil \\ E-mail: rodrigo.rosa@uftm.edu.br \\ Fabrizio Antônio Gomide Cardoso \\ ORCID: https://orcid.org/ 0000-0001-7520-2879 \\ Universidade Federal do Triângulo Mineiro, Brasil \\ E-mail: fabrizio.cardoso@uftm.edu.br \\ Ana Paula Espindula \\ ORCID: https://orcid.org/0000-0002-9282-4482 \\ Universidade Federal do Triângulo Mineiro, Brasil \\ E-mail: ana.espindula@uftm.edu.br
}

\begin{abstract}
Resumo
A Síndrome de Down apresenta alterações biomecânicas e estruturais que resultam em déficit na ativação muscular e consequentemente no equilíbrio. Diante disto, a Realidade Virtual surge como instrumento terapêutico, no qual são apresentadas aos usuários oportunidades de envolvimento em ambientes virtuais, que adiciona um fator motivacional e lúdico à reabilitação. Objetivo: Verificar os efeitos da terapia de Realidade Virtual no equilíbrio e na atividade muscular de membros inferiores de crianças com Síndrome de Down. Métodos: A amostra foi composta por indivíduos com Síndrome de Down com idade média de 10,8 \pm 3,4 anos, submetidos a avaliações motoras pré e pós intervenções em terapia de Realidade Virtual por meio do Nintendo Wii. Resultados: O deslocamento medial e lateral $(\mathrm{p}=0,016)$, deslocamento anteroposterior $(\mathrm{p}=0,056)$ e atividade muscular do músculo tibial anterior $(\mathrm{p}=0,001)$ tiveram melhora significativa após a terapia. Conclusão: A Terapia proposta é eficiente e está indicada para a melhora do equilíbrio e atividade muscular de membros inferiores das crianças com Síndrome de Down.
\end{abstract}

Palavras-chave: Síndrome de Down; Realidade virtual; Equilíbrio postura; Eletromiografia.

\begin{abstract}
Down syndrome has biomechanical and structural changes that result in a deficit in muscle activation and, consequently, in balance. In view of this, Virtual Reality emerges as a therapeutic tool, in which users are presented with opportunities to be involved in virtual environments, which adds a motivational and playful factor to rehabilitation. Objective: To verify the effects of Virtual Reality therapy on the balance and muscle activity of lower limbs of children with Down Syndrome. Methods: The sample consisted of individuals with Down Syndrome with a mean age of $10.8 \pm 3.4$ years, who underwent motor assessments before and after interventions in Virtual Reality therapy using the Nintendo Wii. Results: Medial and lateral displacement $(\mathrm{p}=0.016)$, anteroposterior displacement ( $\mathrm{p}$ $=0.056)$ and muscle activity of the anterior tibial muscle $(\mathrm{p}=0.001)$ had significant improvement after therapy. Conclusion: The proposed therapy is efficient and is indicated for improving the balance and muscle activity of lower limbs of children with Down Syndrome.
\end{abstract}

Keywords: Down Syndrome; Virtual reality; Postural balance; Electromyography. 


\section{Resumen}

El síndrome de Down tiene cambios biomecánicos y estructurales que dan como resultado un déficit en la activación muscular y, en consecuencia, en el equilibrio. Ante esto, la Realidad Virtual surge como una herramienta terapéutica, en la que se presenta a los usuarios oportunidades para involucrarse en entornos virtuales, lo que añade un factor motivacional y lúdico a la rehabilitación. Objetivo: Verificar los efectos de la terapia de Realidad Virtual sobre el equilibrio y la actividad muscular de miembros inferiores de niños con Síndrome de Down. Métodos: La muestra estuvo constituida por individuos con Síndrome de Down con una edad media de 10,8 \pm 3,4 años, a los que se les realizaron evaluaciones motoras antes y después de intervenciones en terapia de Realidad Virtual utilizando la Nintendo Wii. Resultados: El desplazamiento medial y lateral $(\mathrm{p}=0,016)$, el desplazamiento anteroposterior $(\mathrm{p}=$ $0,056)$ y la actividad muscular del músculo tibial anterior $(\mathrm{p}=0,001)$ tuvieron una mejoría significativa después de la terapia. Conclusión: La terapia propuesta es eficaz y está indicada para mejorar el equilibrio y la actividad muscular de los miembros inferiores de los niños con Síndrome de Down.

Palabras clave: Síndrome de Down; Realidad virtual; Equilibrio postural; Electromiografia.

\section{Introdução}

As crianças com Síndrome de Down (SD) apresentam diferentes alterações sensoriais e motoras que convergem em um desenvolvimento de habilidades motoras de forma atípica (Barros \& Fonseca, 2004). Esta condição é caracterizada pela anomalia cromossômica, que se apresenta como uma trissomia do cromossomo 21, fato que provoca desequilíbrio da função reguladora que os genes exercem sobre a síntese de proteína, perda de harmonia no desenvolvimento e nas funções das células, que resultam em alterações fenotípicas e atraso no desenvolvimento motor (Lobe et al., 2013; De Almeida, Dos Santos Moreira \& Tempski, 2013; Borssatti, Dos Anjos \& Ribas, 2017). Fisicamente, estão presentes a hipotonia, a fraqueza muscular, a hipermobilidade articular e a frouxidão ligamentar, fatores que colaboram para um déficit no equilíbrio (Mazzone, Mugno \& Mazzone, 2004; Chiviacowsky et al., 2012).

Crianças com SD são menos eficientes na seleção e utilização de informações sensoriais adequadas ao contexto em que a tarefa é realizada (Meneghetti, 2009). Em decorrência da dificuldade para captar a posição do corpo no espaço e a velocidade em que o corpo está se movendo, as crianças com SD apresentam um maior padrão oscilatório. Isto parece ocorrer principalmente quando há déficits provenientes de algum sistema sensorial, tornando-se ainda maior a oscilação corporal entre indivíduos com SD (Webber, 2004).

Todas estas características supracitadas influenciam negativamente nos dois componentes comportamentais do sistema de controle postural: a orientação e o equilíbrio postural. A orientação postural é o posicionamento relativo do corpo aos demais segmentos e o meio ambiente, ou seja, orientar o corpo para a manutenção vertical e alinhar os segmentos em relação aos demais segmentos para manutenção da posição ereta (Carvalho \& Almeida, 2008). Já o equilíbrio postural é referente à habilidade de manter a posição do corpo dentro dos limites de estabilidade por meio da relação das várias forças que atuam sobre o corpo, como a força da gravidade, dos músculos e a inércia. Pode-se considerar como tarefa básica do equilíbrio a manutenção da estabilidade corporal tanto em condição estática quanto dinâmica (Teixeira, 2013).

As características neurológicas presentes nessa população direcionam os objetivos e condutas a serem realizadas pelos profissionais da saúde durante o processo de desenvolvimento neuropsicomotor, constituindo um pré-requisito para que as crianças possam adquirir as habilidades sensoriais, cognitivas e motoras (Siqueira \& Gurgel-Giannetti, 2011).

Especialmente no caso de crianças com SD é importante considerar alternativas terapêuticas interessantes e lúdicas, como método diferencial. Nesse contexto, tecnologias inovadoras surgem a cada dia trazendo a possibilidade de uma otimização das condutas no âmbito da reabilitação. Diante disto, a Realidade Virtual (RV) surge como um recurso promissor no tratamento fisioterapêutico, com resultados benéficos no âmbito da reabilitação, pois é capaz de incentivar a criança com SD e também adultos, deixando-os mais motivados e concentrados nas atividades, bem como favorecendo a aquisição de suas habilidades motoras, resistência muscular, equilíbrio (Junior \& Da Silva, 2012; Medeiros, 2013; Monte et al., 2020). Durante a 
realização das tarefas, o paciente treina habilidades de planejamento e controle motor, cria estratégias para vencer seus desafios motores, e assim estimula a plasticidade do sistema nervoso central (Steindl et al., 2006).

O presente estudo teve como objetivo avaliar os benefícios da terapia de RV no equilíbrio e na atividade elétrica muscular de membros inferiores de crianças com SD. Tendo como hipótese de que a terapia de RV proporciona melhora no equilíbrio e aumento da atividade elétrica muscular de cadeias musculares envolvidas para a realização dos jogos.

\section{Metodologia}

Estudo de série de casos do tipo quantitativo e longitudinal (Pereira et al., 2018), submetido e aprovado pelo Comitê de Ética em Pesquisa, sob parecer 3.034.675/2018.

Os pais e/ou responsáveis pelas crianças receberam esclarecimentos quanto aos objetivos e procedimentos a serem realizados pelo estudo e aqueles que consentiram a participação de seu tutelado assinaram o Termo de Consentimento Livre e Esclarecido e de Assentimento. Posteriormente a autorização, os participantes da pesquisa ingressaram nos procedimentos metodológicos no período de fevereiro a julho de 2019.

A amostra teve início com oito indivíduos com diagnóstico de SD (Trissomia simples), porém no decorrer dos atendimentos houve perda amostral de três sujeitos, que apresentaram dificuldade em compreender os comandos e tarefas realizadas durante o atendimento em RV. Assim o estudo finalizou com três indivíduos do sexo masculino e dois do feminino, com idade média de 10,8 $\pm 3,4$ anos, selecionados de acordo com os critérios de inclusão, não inclusão e exclusão, descritos abaixo.

Para os critérios de inclusão, foram utilizadas as informações contidas nos prontuários da Associação de Pais e Amigos dos Excepcionais (APAE) de Uberaba, Minas Gerais para seleção dos participantes da pesquisa. Os indivíduos deveriam ter idade entre oito e quinze anos, de ambos os sexos, regularmente matriculados na APAE/Uberaba-MG, com o Termo de Consentimento Livre e Esclarecido e Termo de Assentimento assinados pelos pais e/ou responsáveis, capacidade de entendimento para os comandos verbais simples, diagnóstico clínico de SD - Trissomia Simples.

Não foram incluídos nesse estudo os indivíduos que já haviam realizado terapia de realidade virtual previamente, impossibilitados de permanecer na posição ortostática, com comprometimento visual grave ou não compensado com uso de lentes corretivas e crianças com distúrbios ortopédicos que resultavam em quadro álgico.

Para avaliação do equilíbrio, os participantes foram avaliados por dois instrumentos, o Teste Clínico de Integração Sensorial e Equilíbrio (CTSIB) e o sistema Polhemus.

O CTSIB foi desenvolvido com objetivo de identificar a contribuição dos três principais sistemas sensoriais: visão, vestibular e somatossensorial, envolvidos no equilíbrio (Riley \& Clark, 2003; Steindl et al., 2006) e busca isolar as diversas contribuições sensoriais (Tabela 1) por meio da remoção ou distorção da superfície ou da visão (Steindl et al., 2006; Wrisley, 2007). Os participantes foram instruídos a ficarem com os braços relaxados ao lado do corpo, pés posicionados lado a lado a uma distância semelhante a dos ombros e com a cabeça ereta. Nas condições com os olhos abertos foi solicitado que as crianças olhassem em um marcador circular com um diâmetro de $5,5 \mathrm{~cm}$ fixado na parede em frente ao participante a uma distância de $150 \mathrm{~cm}$. O participante deveria manter-se pelo tempo de 30 segundos em cada uma das condições, sendo realizadas três medidas e levada em consideração a média destas. Para padronização do solo instável, foi utilizado uma espuma de densidade de $28 \mathrm{~g} / \mathrm{cm}^{3}$, com 7,5 $\mathrm{cm}$ de altura, $50 \mathrm{~cm}$ de comprimento e $50 \mathrm{~cm}$ de largura (Shumway-Cook \& Woollacott, 2003), (Tabela 1). 
Tabela 1: Condição para Teste Clínico de Integração Sensorial de Equilíbrio (CTSIB).

\section{Condição 1 \\ Condição 2 \\ Condição 3 \\ Condição 4 \\ Condição 5}

Condição 6

olhos abertos, pés juntos, em superfície estável

olhos fechados com venda, pés juntos, em superfície estável

olhos abertos, com pés juntos, sobre superfície instável

olhos fechados, com pés juntos, sobre superfície instável

olhos abertos, pés em posição de Tandem (calcanhar do pé não dominante posicionado logo à frente do hálux do pé dominante) em superfície instável

olhos abertos, em posição unipodal (membro dominante) em superfície instável

Fonte: Autores.

O sistema Polhemus (POLHEMUS 3SPACE® ISOTRAK II, Colchester, Canada) foi utilizado para avaliar o equilíbrio estático e dinâmico, com objetivo de mensurar a máxima amplitude de deslocamento de tronco no plano anteroposterior. Este sistema é composto por sensores eletromagnéticos, que se baseiam na emissão e detecção de campo magnético, onde uma bobina transmissora capta a oscilação postural por meio de um sensor posicionado no corpo do indivíduo; sendo conectada a um amplificador. Os dados obtidos foram transferidos para um computador em tempo real por meio da interface USB (Universal Serial Bus) e de um software desenvolvido em ambiente Labview. Para a realização da avaliação, a bobina transmissora foi posicionada em um suporte próximo ao indivíduo avaliado (distância de $60 \mathrm{~cm}$ ) e a bobina sensora fixada na sétima vértebra cervical (C7) com fita adesiva (Guerraz, 2000).

Para a avaliação do equilíbrio estático os participantes foram avaliados durante 90 segundos nas quatro primeiras condições do teste CTSIB descritas na Tabela 1. Na avaliação dinâmica o avaliador posicionou-se atrás da criança para segurar a cadeira e evitar que ela sentasse no fio do sensor e posteriormente foi solicitado que as crianças realizassem o teste de levantar e sentar cinco vezes consecutivas, neste, foram utilizadas diferentes alturas de bancos para que o joelho das crianças se mantivesse em noventa graus de flexão enquanto sentada, além disso, as crianças foram orientadas a posicionarem as mãos cruzadas sobre o tórax para evitar qualquer auxilio de membros superiores ao se levantar. As variáveis analisadas foram o tempo gasto para a realização do teste e o valor médio do deslocamento máximo anteroposterior e médio - lateral de tronco. Para a análise dos dados obtidos foi utilizado o software Origin 6.0 (Guerraz, 2000).

Para análise eletromiográfica (EMG) utilizou-se o Eletromiógrafo de Superfície portátil, modelo EMG800RF da marca EMG System do Brasil Ltda®, de oito canais via wireless. Os eletrodos foram posicionados segundo as recomendações do Projeto SENIAM (Surface ElectroMyoGraphy for Non-invasive Assessment of Muscles), em musculatura de membros inferiores bilateralmente: Reto Femoral, Vasto Lateral, Vasto Medial e Tibial Anterior, após a realização da tricotomia e limpeza do local com algodão embebido em álcool a 70\% a fim de facilitar a aderência na pele e diminuir a impedância dos sinais. Utilizamos eletrodos do tipo descartáveis bipolares ativos de superfície de $\mathrm{Ag} / \mathrm{AgCl}$ de espuma e gel sólido autoadesivo de $1 \mathrm{~cm}$ em forma de disco conectados aos pré-amplificadores com distância de centro a centro de 2 cm de diâmetro, segundo as recomendações da International Society of Electrophysiology and Kinesiology (ISEK) (Bevilacqua Junior, 2018 \& Espindula et al., 2014).

Os registros eletromiográficos foram coletados antes e após o primeiro e décimo atendimento nas condições de repouso, em pé, estável, sobre a plataforma inicial (PPI) e na mesma condição na avaliação final (plataforma final (PPF). E, em condição dinâmica (1`,15` e 30` minutos da intervenção). Para análise das condições em repouso foi utilizado o valor de RMS (Root Mean Square) bruto, enquanto para as análises das condições dinâmicas usamos o valor integrado do sinal. Os participantes incluídos no estudo não apresentaram capacidade de compreensão para realização da contração voluntária máxima para que os sinais fossem normalizados. 
As avaliações e atendimentos na terapia de RV utilizando Nintendo® Wii foram realizadas na APAE de Uberaba/MG, em uma sala específica e adequada para o desenvolvimento das atividades, tendo as intervenções a frequência de uma vez por semana, durante 30 minutos o que totalizou 10 atendimentos. Os jogos WiiFit juntamente com o acessório plataforma Balance Board, foram utilizados como ferramentas para o presente estudo. Foram selecionados quatro jogos inseridos nas categorias força e equilíbrio (Tabela 2). Cada partida de um jogo demorava cerca de 3 minutos e eram repetidos ciclicamente até atingir um tempo total de 30 minutos de terapia. No decorrer das intervenções o participante recebeu explicações verbais e visuais por meio de modelo de como jogar cada um dos jogos.

Tabela 2: Detalhamento dos jogos utilizados.

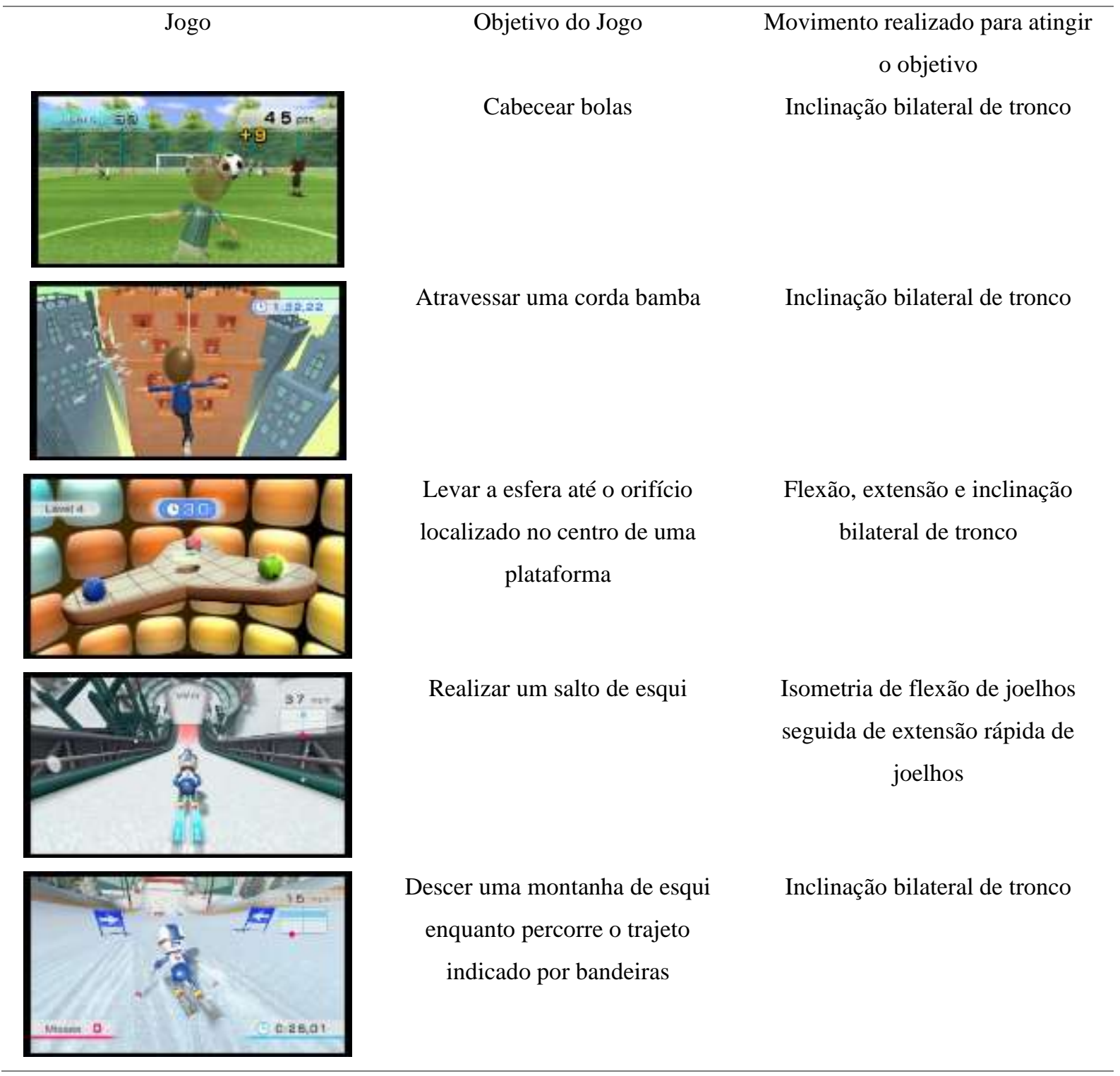

Fonte: <https://www.nintendo.pt/index.html>

Para análise estatística foram elaboradas planilhas eletrônicas por meio do programa Microsoft Excelß, onde os dados foram analisados. A normalidade dos dados foi verificada a partir do teste de Shapiro Wilk e a homogeneidade das variâncias pelo teste de Bartlett. As variáveis contínuas que apresentaram distribuição normal foram expressas em média e desvio padrão $( \pm)$ e aquelas com distribuição não normal foram expressas em mediana, com valores máximos, mínimos e percentis. Nas comparações entre pré e pós intervenção em terapia de RV (10 atendimentos), as variáveis que apresentaram distribuição 
normal e variância homogênea foram analisadas pelos testes " $\mathrm{t}$ " não pareado e as amostras repetidas "t" pareado, enquanto aquelas com distribuição não normal, ou mesmo normal, porém com variáveis não homogêneas utilizou-se o teste de MannWhitney. Nas análises de múltiplas variáveis, utilizou o Teste Anova pareado seguido do pós hoc de Tukey para distribuição normal, e para os dados não normais, o Teste de Kruskall-Wallis seguido do pós-hoc de Dunn. Foram consideradas estatisticamente significativas as diferenças em que a probabilidade foi menor que $5 \%$, ou seja, $\mathrm{p}<0,05$.

Nos resultados que não apresentaram diferenças estatisticamente significativas foi aplicado o teste $\mathrm{D}$ de Cohen para verificarmos se os resultados, apesar de não significativos estatisticamente, apresentavam relevância clínica.

\section{Resultados}

No Teste CTSIB, as condições 1, 2 e 3 foram concluídas em 100\% dos participantes, atingindo 30 segundos antes e após as intervenções. Na condição 4, 60\% concluíram o teste antes das intervenções, e as demais crianças permaneceram em média por 16,5 $\pm 1,31$ segundos. Posteriormente às intervenções $100 \%$ dos participantes concluíram a condição 4 . Ao analisar a condição 5 , anteriormente às intervenções, $80 \%$ não completaram essa condição permanecendo em média por $7,25( \pm 4,42)$ segundos, e 20\% (uma criança) foi capaz de concluí-la. Entretanto, após a terapia de RV, $80 \%$ concluíram o teste, e uma criança manteve-se incapaz de concluí-lo e manteve-se na condição por 20 segundos. Por último, a sexta condição não foi concluída em $100 \%$ dos participantes, mesmo após as intervenções, porém, houve um aumento na média de tempo alcançada antes da terapia ( $3,8 \pm 2,94$ segundos) comparado com pós intervenção ( $11 \pm 4,52$ segundos).

Segundo a análise por meio do teste D de Cohen, as condições 5 (d: -5,01) e 6 (d: -2,33) apresentaram grande diferença e alta relevância clínica, na comparação antes e depois intervenção com RV.

Os resultados obtidos por meio do Polhemus, demonstraram diferença estatística entre pré e pós intervenção com RV no teste dinâmico de sentar e levantar (cadeira) no deslocamento medial-lateral $(\mathrm{p}=0,016)$, velocidade média nos sentidos ântero-posterior $(\mathrm{p}=0,010)$ e medial-lateral $(\mathrm{p}=0,008)$ (Figura 1$)$.

Figura 1: Teste dinâmico de Sentar e levantar da cadeira utilizando o Polhemus para avaliação em Cm dos deslocamentos: (1a): Ântero-posterios (AP), médial-lateral (ML) e (1b): Velocidade média AP e ML.

\section{Teste dinâmico de Sentar e levantar da cadeira}
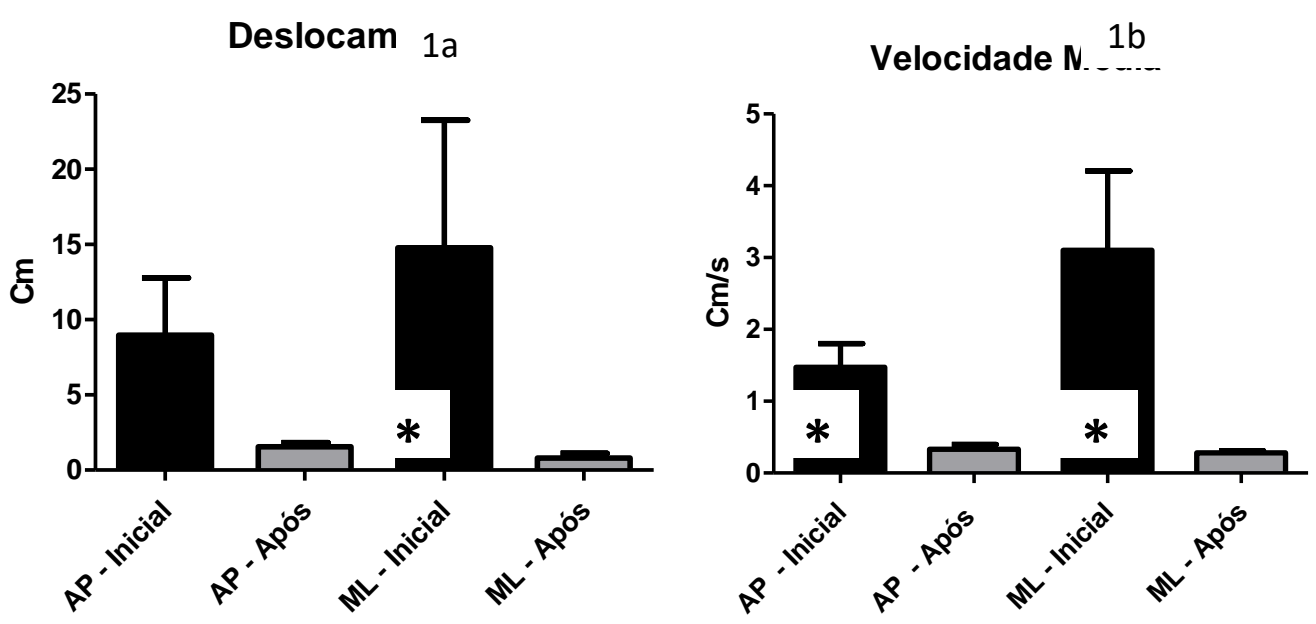

Fonte: Autores. 
Ainda no Polhemus, o teste D de Cohen indicou alta relevância clínica na comparação dos seguintes momentos: sentar e levantar na velocidade média anteroposterior $(\mathrm{d}=-1,12)$, e na velocidade média medial-lateral $(\mathrm{d}=-1,81)$. Condição 1 na área de elipse $(d=1,16)$, na frequência média anteroposterior $(d=-0,90)$ e na frequência média medial-lateral $(d=-1,33)$. Condição 2 no deslocamento anteroposterior $(\mathrm{d}=1,32)$, no deslocamento medial-lateral $(\mathrm{d}=1,13)$, e na frequência media anteroposterior $(\mathrm{d}=-1,58)$. Condição 3 na velocidade média medial - lateral $(\mathrm{d}=-0,88)$, no deslocamento da trajetória $(\mathrm{d}=1,24)$, e na frequência média medial - lateral $(\mathrm{d}=-0,85)$. Condição 4 não apresentou valores relevantes pelo teste $\mathrm{D}$ de Cohen.

Os estatocinesiogramas apresentados na Figura 2, representam graficamente o deslocamento medial-lateral e ânteroposterior pré e pós intervenções de um paciente, com o objetivo de exemplificar a diminuição da amplitude de deslocamento após a terapia de RV.

Figura 2: Estatocinesiograma do deslocamento Ântero-Posterior e Medio-Lateral de um paciente antes e após 10 atendimentos no Wii: (1a) cadeira, antes dos atendimentos; (1aa) cadeira, após os atendimentos; (1b) Olhos abertos em posição de bipedestação em superfície de espuma antes dos atendimentos; (1bb) Olhos abertos em posição de bipedestação em superfície de espuma após atendimentos; (1c) Olhos fechados em posição de bipedestação em superfície de espuma antes dos atendimentos; (1cc) Olhos fechados em superfície de espuma após atendimentos; (1d) Olhos abertos em posição de bipedestação em superfície chão de piso antes dos atendimentos; (1dd) Olhos abertos em posição de bipedestação em superfície chão de piso após atendimentos; (1e) Olhos fechados em posição de bipedestação em superfície chão de piso antes dos atendimentos; (1ee) Olhos fechados em posição de bipedestação em superfície chão de piso após atendimentos. Fonte: Software Polhemus.

\section{Estatocinesiograma do deslocamento Ântero-Posterior e Medio-Lateral}
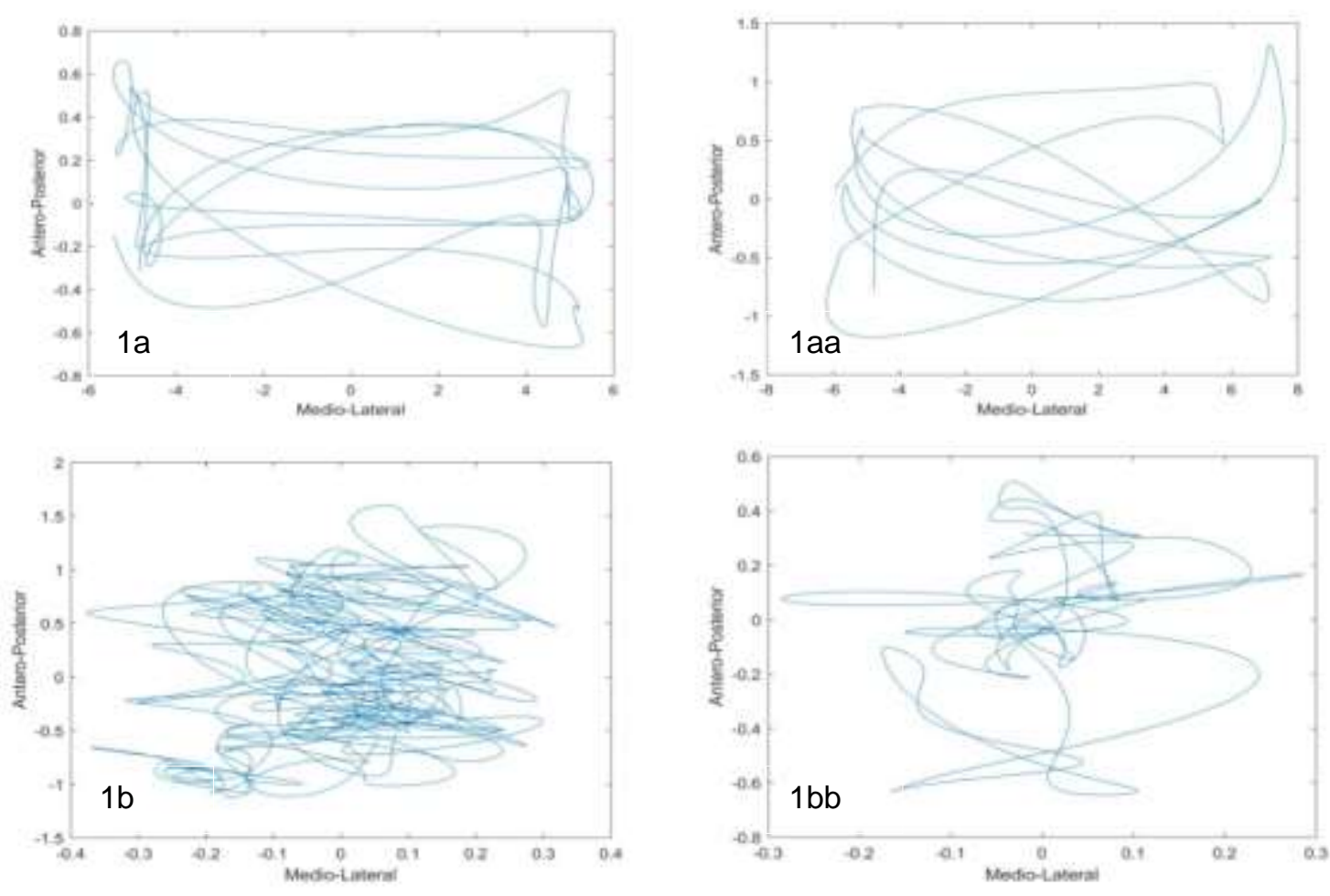

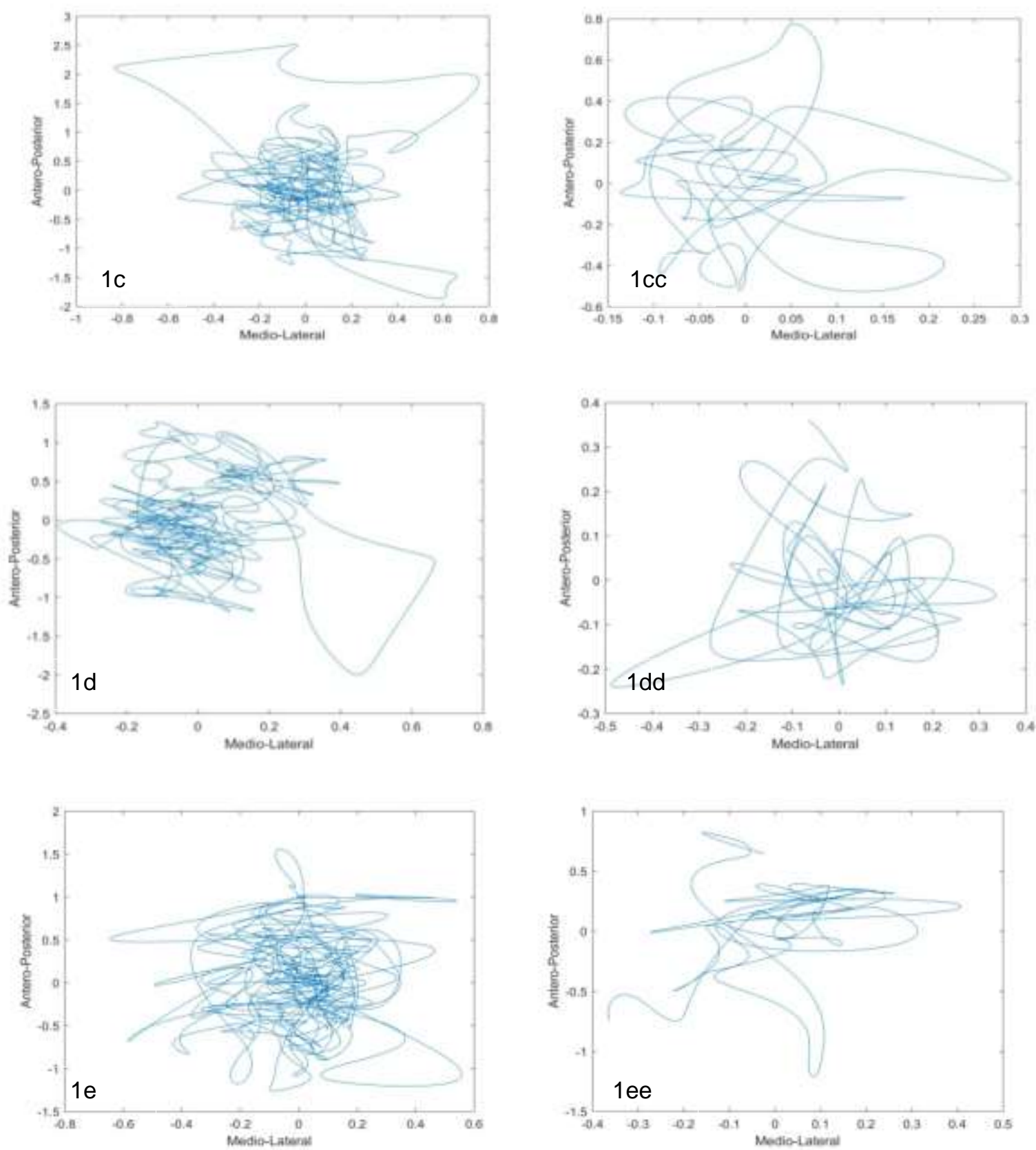

Fonte: Autores.

As análises por meio da EMG apresentaram aumento significativo da atividade elétrica do músculo tibial anterior esquerdo no 30 minuto do décimo atendimento quando comparado ao ao PPI do primeiro atendimento $(\mathrm{p}=0,001)$ e ao primeiro minuto do décimo atendimento $(\mathrm{p}=0,001)$. Já o tibial anterior direito apresentou aumento da atividade elétrica muscular na posição PPI do primeiro atendimento comparado ao primeiro $(\mathrm{p}=0,001)$ e trigéssimo $(\mathrm{p}=0,001)$ minuto do décimo atendimento (Figura 3). 
Figura3: Dados eletromiográficos do músculo tibial anterior direito e esquerdo.

\section{Eletromiografia do músculo Tibial anterior}
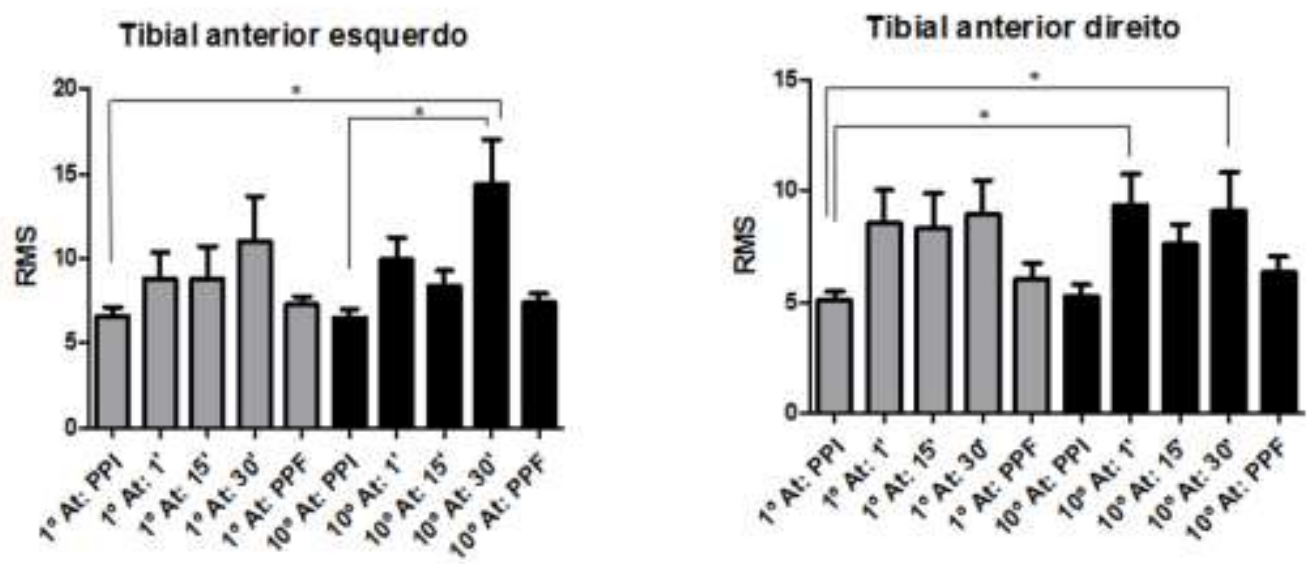

Fonte: Autores.

\section{Discussão}

O presente estudo teve como objetivo avaliar os benefícios da terapia de RV no equilíbrio e na atividade elétrica muscular de membros inferiores de crianças com SD. Sendo nossa hipótese validada, pois houve melhora da atividade elétrica de um músculo, assim como melhora do equilíbrio estático e dinâmico dos sujeitos avaliados.

Em uma revisão bibliográfica voltada exclusivamente para aplicação da RV como instrumento da Fisioterapia em crianças com SD, foram encontrados estudos que apontaram diferentes benefícios obtidos com a RV (De Carvalho Mello \& Ramalho, 2015). Dentre estes, um relato de caso envolvendo uma criança de doze anos com SD, foi observado que o equilíbrio pode sofrer influências significativas da terapia com melhora nos testes de oscilação, de controle postural, equilíbrio, agilidade e velocidade (Berg et al., 2012). Fato que coincide com os resultados encontrados neste estudo, no qual as variáveis relacionadas com o equilíbrio (CTSIB e Polhemus) apresentaram melhoras significativas, tendo em vista a utilização de escalas de níveis de sensibilidade para esta população, os resultados convergem para o mesmo desfecho, melhora do equilíbrio estático e dinâmico.

O ganho de equilíbrio, frequentemente observado nas crianças após atividade com o videogame está correlacionado à possibilidade proporcionada pelo método de favorecer tanto o equilíbrio estático, quanto o dinâmico, diferente do que se percebe em outras técnicas fisioterapêuticas, que têm como foco, em alguns momentos, o equilíbrio estático (Mombarg, Jelsma \& Hartman, 2013. Neste ponto, é possível perceber que o resultado encontrado no software Polhemus durante a avaliação do equilíbrio dinâmico (sentar e levantar da cadeira), se justifica pelo maior enfoque da RV no treino de equilíbrio dinâmico comparado a outras técnicas no âmbito da reabilitação.

Análises da atividade elétrica muscular de membros inferiores de crianças com SD por meio da EMG durante intervenções terapêuticas em RV não foram encontradas na literatura, no entanto, o presente estudo observou que durante alguns momentos da terapia com RV crianças com SD apresentaram maior atividade do músculo tibial anterior bilateralmente ao comparar com os momentos de repouso (PPI). Esse resultado pode estar relacionado com o fato de que os indivíduos com SD realizam ativação muscular simultânea de músculos agonistas e antagonistas mesmo em movimentos mais simples (Latash, 
1992; Ferreira, 2000) isso acontece para proporcionar maior estabilidade articular durante os movimentos, já que a musculatura dessa população tem característica hipotônica (Latash, 1992).

Além disso, as características da SD também podem interferir no desenvolvimento das noções temporais, devido à presença de diferenças neuro anatômicas e funcionais no cérebro destas crianças. De acordo com a literatura, na SD todos os neurônios formados têm sua organização, estrutura e funcionamento afetados, tais alterações afetam o desenvolvimento dos circuitos cerebrais, influenciando sobre os mecanismos da atenção, memória, capacidade de correlação e análise, pensamento abstrato, entre outros (Flórez \& Troncoso, 1997). Fato que justifica a necessidade de supervisionamento constante dessa população enquanto realiza terapia de RV.

Estudos apontaram melhora na motricidade global, equilíbrio, esquema corporal e organização espacial de indivíduos com SD, após 20 atendimentos de terapia de RV, fato que corrobora com os resultados do presente estudo, sugerindo ainda que neste, dez atendimentos foram suficientes para alcançar melhora no âmbito do equilíbrio corporal e atividade elétrica muscular de membros inferiores (Lorenzo, Braccialli \& Araújo, 2015; Andrade, Moreira \& Brandão, 2020). Nesse sentido, torna-se evidente a necessidade de propor intervenções que estimulem os aspectos do desenvolvimento que podem estar prejudicados pelas características inerentes à SD trazendo um componente lúdico e motivador.

Os benefícios relacionados a melhora do equilíbrio e da atividade muscular de membros inferiores advindos da terapia com a RV podem auxiliar na modificação do estilo de vida sedentário, comumente observado na população com SD em decorrência do alto gasto energético exigido a estes indivíduos para realização de atividades e da consequente baixa tolerância ao exercício físico.

Limitações do estudo: Os resultados obtidos por meio desse estudo não podem ser generalizados para todas as pessoas com SD. Ressaltamos a importância do estudo em um maior número de indivíduos. Além disso, o período de tempo em que os achados podem ser mantidos precisa ser avaliado em testes de acompanhamento.

\section{Conclusão}

Concluímos, portanto, que a terapia de RV foi capaz de resultar em melhoras no equilíbrio estático e dinâmico, bem como a ativação elétrica do músculo tibial anterior bilateralmente de crianças com SD. Os achados inferem que a RV é uma forma benéfica e lúdica de terapia para crianças com SD. Para trabalhos futuros sugere-se que seja realizado estudo com maior número de participantes e que os resultados alcançados sejam acompanhado ao longo do tempo.

\section{Referências}

Andrade, G. de O., Moreira, S. A. S., Brandão, N. S. (2020). Aprendizagem por meio de jogo educativo: uma experiência com crianças, jovens e adultos com síndrome de Down. Research, Society and Development, 9(6), e 07963309. 10.33448/rsd-v9i6.3309.

Barros, D \& Fonseca, V. (2004). Psicomotricidade. Perspectivas Multidisciplinares. Porto Alegre, Artes Médica. Levin EA. Clínica Psicomotora. Petrópolis: Vozes, 1995a. Levin E A Infância em Cena. Petrópolis. Vozes, 1995b. _-artigo "À margem da Infância-um estudo. In: IV Congresso Internacional Ex Seminário Nacional Do Ines. p. 107.

Berg, P., Becker, T., Martian, A., Danielle Primrose, K \& Wingen, J. (2012). Motor control outcomes following Nintendo Wii use by a child with Down syndrome. Pediatric Physical Therapy, 24 (1), 78-84.

Bevilacqua Junior, D. E. (2018). Avaliação cardiovascular, muscular e da mobilidade funcional de adolescentes com síndrome de Down atendidos na Equoterapia e Fisioterapia. Tese de doutorado. Universidade Federal do Triângulo Mineiro.

Borssatti, F., Dos Anjos, F. B \& Ribas, D. I. R. (2017). Efeitos dos exercícios de força muscular na marcha de indivíduos portadores de Síndrome de Down. Fisioterapia em Movimento, 26 (2).

Carvalho, R. L \& Almeida, G. L. (2008). Controle postural em indivíduos portadores da síndrome de Down: revisão de literatura. Fisioterapia e Pesquisa, v. $15(3), 304-308$.

Chiviacowsky, S., Wulf, G., Machado, C \& Rydberg, N. (2012). Self-controlled feedback enhances learning in adults with Down syndrome. Brazilian Journal of Physical Therapy, 16(3), 191-196. 
De Almeida, M. D., Dos Santos Moreira, M. C \& Tempski, P. Z. (2013). A intervenção fisioterapêutica no ambulatório de cuidado a pessoa com síndrome de Down no Instituto de Medicina Física e Reabilitação HC FMUSP. Revista Acta Fisiatrica, 20 (1), 55-62.

De Carvalho Mello, B. C \& Ramalho, T. F. (2015). Uso da realidade virtual no tratamento fisioterapêutico de indivíduos com Síndrome de Down. Revista Neurociências, 23 (1), 143-149.

Dias, R. S., Sampaio Italo, L. A \& Taddeo, L. S. (2009). Fisioterapia X Wii: a introdução do lúdico no processo de reabilitação de pacientes em tratamento fisioterápico. In: VIII Brazilian Symposium on Games and Digital Entertainment. 8-10.

Espindula, A. P., Ribeiro, M. F., Sande de Souza, L. A. P., Ferreira, A. A., Ferraz, M. L. F \& Teixeira, V. P. A. (2014). Riding equipment for hippotherapy in individuals with Down syndrome: an electromyographic study. ConScientiae Saúde, 13 (3), 349.

Ferreira, S. M. S. (2000). Modulação da latência da musculatura antagonista em indivíduos neurologicamente" normais" e portadores da síndrome de Down. Tese de Doutorado. Instituto de Biociências do Campus de Rio Claro da Universidade Estadual Paulista.

Flórez, B. J \&Trancoso, V.M. (1997). Síndrome de Down y educacíon. Barcelona: Masson - Salvat Medicina y Santander.

Guerraz, M., Sakellari, V., Burchill, P \& Bronstein, A. M. (2000). Influence of motion parallax in the control of spontaneous body sway. Experimental brain research, 131 (2), 244-252.

Junior, R. S. M \& Da Silva, E. B. (2012). Virtual rehabilitation effectiveness in the body balance and motor skills of individuals with neuromotor deficit: a systematic review. Revista Brasileira de Atividade Física \& Saúde, 17 (3), 224-230.

Latash, M. L. (1992). Motor control in Down syndrome: The role of adaptation and practice. Journal of Developmental and Physical Disabilities, v. 4 (3), $227-261$.

Lobe, M. C. S., Perini, L. D. P., Noronha, M. G. O., Krueger, M. B \& Castellen, N. R. C. (2013). Prevalência de doenças autoimunes em pacientes com síndrome de Down. Revista da AMRIGS, 57 (1), 5-8.

Lorenzo, S. M. de., Braccialli, L. M. P \& Araújo, R. C. T. (2015). Realidade virtual como intervenção na síndrome de Down: uma perspectiva de ação na interface saúde e educação. Revista Brasileira de Educação Especial, 21 (2), 259-274.

Mazzone, L., Mugno, D \& Mazzone, D. (2004). The General Movements in Children with Down Syndrome. Early Human Development. 79 (2), 119-30.

Medeiros, D. (2013). Uma ferramenta de interação 3D baseada em tablet para ambientes de engenharia virtual. In: Anais da $12^{\text {a }}$ Conferência Internacional ACM SIGGRAPH sobre Continuum de Realidade Virtual e Suas Aplicações na Indústria. 211-218.

Meneghetti, C. H. Z., Blascovi-Assis, S. M., Deloroso, F. T \& Rodrigues, G. M. (2009). Avaliação do equilíbrio estático de crianças e adolescentes com síndrome de Down. Brazilian Journal of Physical Therapy, 13 (3), 230-235.

Mombarg, R., Jelsma, D \& Hartman, E. (2013). Effect of Wii-intervention on balance of children with poor motor performance. Research in developmental disabilities, 34 (9), 2996-3003.

Monte, J. A., Gomes, V. M. DA S. A., Silveira, T. M. V. DA., Arruda, L. Q., Carvalho, V. C. P., Barros, M. L. N., Uchôa Érica P. B. L. (2020). Efeito do uso da reabilitação virtual com o X-box® no risco de quedas em idosos. Research, Society and Development, 9 (10), e3049108638. 10.33448/rsd-v9i10.8638.

Pereira, A. S., Shitsuka, D. M., Parreira, F. J \& Shitsuka, R. (2018). Metodologia da pesquisa científica. UFSM.https://repositorio.ufsm.br/bitstream/handle/1/15824/Lic_Computacao_Metodologia-Pesquisa-Cientifica.pdf?sequence=1

Riley Michael, A \& Clark, S. (2003). Recurrence analysis of human postural sway during the sensory organization test. Neuroscience Letters, 342, (1), 45-48.

Shumway-Cook, A \& Woollacott, M. H. (2003). Controle motor: teoria e aplicações práticas. Manole.

Siqueira, C. M \& Gurgel-Giannetti, J. (2011). Mau desempenho escolar: uma visão atual. Revista da Associação Médica Brasileira, 57 (1), $78-87$.

Steindl, R., Kunz, K., Schrott-Fischer, A \& Scholtz, A. W. (2006). Effect of age and sex on maturation of sensory systems and balance control.

Developmental medicine and child neurology, 48 (6) 477-482.

Teixeira, C. L. (2013). Equilíbrio e controle postural. Sistema eletrônico de editoração de revistas. 11(20).

Webber, A., Virji-Babul, N., Edwards, R \& Lesperance, M. (2004). Stiffness and postural stability in adults with Down syndrome. Experimental Brain Research, 155(4), 450-458.

Wrisley, D. M., Stephens, M. J., Mosley, S., Wojnowski, A., Duffy, J \& Burkard, R. (2007). Learning effects of repetitive administrations of the sensory organization test in healthy young adults. Archives of physical medicine and rehabilitation, 88 (8), 1049-1054. 